\title{
Perspectives on the Emerging Digital Transformation of the Management of Psychiatric Comorbidities in Chronically III Populations
}

Kim Peter Norman, $M D^{1^{*}}$, Seth R Norman, MBA, MAPP2, Mahima Muralidharan, Psy $D^{2}$, Fikry Isaac, MD, MPH ${ }^{2}$, Mary Anne North ${ }^{3}$, Dexter W Shurney, MD, MBA, MPH, FACLM, DipABLM ${ }^{4}$, Dustin W Kieschnick, PsyD ${ }^{1}$ and Kimberlie L Cerrone, MS, MBA, JD2

${ }^{1}$ Department of Psychiatry, UCSF Young Adult and Family Center, UCSF Weill Institute for Neurosciences, University of California, San Francisco, USA

${ }^{2}$ Tiatros Inc, USA

${ }^{3}$ Polaris Management, Inc, USA

${ }^{4}$ American College of Lifestyle Medicine, USA

\begin{abstract}
Chronic mental health and medical conditions are widespread problems that often co-occur. More than $68 \%$ percent of adults with a mental health condition report also having at least one medical condition, and $29 \%$ with a medical condition reported also having a comorbid mental health condition. This perspective manuscript seeks to highlight the interrelatedness of chronic medical and mental health conditions and to discuss emergent digital solutions that can potentially provide comprehensive solutions to the problems of access to mental health care, scalability, and integration of medical and mental health services. Both mental health and medical symptoms impact psychosocial functioning and/or lead to maladaptive behaviors, and this is most often seen in the context of adherence to treatment. The medical conditions of diabetes and cardiovascular disease and their co-occurrences with depression, anxiety, and Posttraumatic Stress Disorder (PTSD) are explored as illustrations of this relationship. Despite the co-occurrence and the inherent treatability of depression, anxiety, and PTSD, there remain significant barriers to access and profound lack of integration between medical and mental health treatments. Advancements in technology provide an encouraging potential solution to problems of access, scalability, and integration. These advancements have come in waves, and we are currently cresting the third wave of digital behavioral health technologies and technology-enabled access. In the third wave of digital behavioral health technologies, the trend is towards addressing underlying mental health conditions and medical comorbidities by unifying the many factors that influence mental and physical health together under a single point of access, integrating the best aspects of individual and group psychotherapy. This third wave of digital delivery of evidence-based mental healthcare programs will open up the possibility that businesses, schools, and even primary care settings can become the opportune point-of-care for mental healthcare and integrated rehabilitation and treatment services. The third wave of digital delivery of evidence-based mental healthcare has the capacity to treat, on a massive scale, the underlying traumas that
\end{abstract}

result in high Adverse Childhood Experiences (ACE) scores, predisposing individuals to variety of chronic medical conditions, such as cardiovascular disease, diabetes, and cancer.

\section{Keywords}

Psychiatric comorbidity, Digital psychiatric therapeutics, Adherence, Mental wellbeing, Wellness, Depression, Anxiety, Holistic care, PTSD, Adverse childhood experiences (ACEs)

\section{Introduction}

Chronic medical illness and mental health conditions are widespread problems with debilitating effects on those whom they afflict. As of 2018 , almost $45 \%$ of Americans had a chronic disease, defined as a disease lasting a year or longer [1]. Almost a third of the U.S. population live with multiple chronic conditions. Medical conditions of heart disease, cancer, and stroke account for more than half of deaths each year, yet people with chronic medical conditions receive a little over half of the recommended preventative health care services [2]. Among mental health conditions, depression, anxiety, and posttraumatic stress disorder (PTSD) have disabling ef-

*Corresponding author: Kim P. Norman, MD, UCSF Distinguished Professor for Adolescent and Young Adult Health, Founder and Director, Department of Psychiatry, UCSF Young Adult and Family Center, UCSF Weill Institute for Neurosciences, University of California, San Francisco, USA and Advisor to Tiatros, Inc.

Accepted: April 13, 2020

Published online: April 15, 2020

Citation: Norman KP, Norman SR, Muralidharan M, et al. (2020) Perspectives on the Emerging Digital Transformation of the Management of Psychiatric Comorbidities in Chronically III Populations. J Psychiatry Treat Res 2(1):25-31 
Citation: Norman KP, Norman SR, Muralidharan M, et al. (2020) Perspectives on the Emerging Digital Transformation of the Management of Psychiatric Comorbidities in Chronically III Populations. J Psychiatry Treat Res 2(1):25-31

fects on tens of millions of Americans each year [3]. A study on the effects of traumatic experiences in childhood, or adverse childhood experiences (ACEs), found that people with high ACE scores were at twelve times higher risk for cardiovascular disease and diabetes, among other medical conditions [4-6]. This perspective manuscript seeks to highlight the interrelatedness of chronic medical and mental health conditions and to discuss emergent digital solutions that can potentially provide comprehensive solutions to the problems of access to mental health care, scalability, and integration of medical and mental health services.

\section{The Interrelated Problem of Chronic Psychi- atric and Medical IIIness}

Mental health and medical conditions often co-occur. In fact, according to analysis of the National Comorbidity Survey, more than $68 \%$ percent of adults with a mental health condition reported also having at least one medical condition, and $29 \%$ with a medical condition reported also having a comorbid mental health condition [7]. A literature review from the Robert Wood Johnson Foundation found that having one type of condition (mental or medical) can be a risk factor for developing the other [8]. Mental health conditions of depression, anxiety, and PTSD disproportionally affect people with chronic medical conditions, including dementia, cardiovascular, cerebrovascular, diabetes, cancer and Parkinson's patients, all of whom have much higher rates of depression, anxiety, and PTSD than found in the general U.S. population [3]. For example, up to $20 \%$ of cardiovascular disease (CVD) patients have Major Depressive Disorder (MDD); and up to $40 \%$ have elevated depressive symptoms [9]. Between 20 to $30 \%$ of patients with CV disease have symptoms of PTSD from exposure to trauma [10-12]. Anxiety is even more common among people with CVD; over $50 \%$ of patients with heart failure have elevated rates of anxiety, and $13 \%$ meet criteria for an anxiety disorder [9]. Similarly, people diagnosed with cancer and cancer survivors are also disproportionally affected by depression and anxiety. One in four cancer patients are diagnosed with clinical depression [13]. Half of all cancer patients report that they feel some anxiety; one-fourth of all people with cancer say they are greatly affected by anxiety [13]. Furthermore, many cancer survivors continue to experience debilitating emotional distress that meets the clinical criteria for anxiety disorder or major depression [13].

Although mental health conditions and medical conditions commonly co-occur, there is no consensus on whether origin of onset begins with one or the other. Results of extant studies suggest a bi-directional relationship and sharing of several risk factors [8]. Risk factors such as ACEs, chronic stress, and socioeconomic factors, including low SES (socioeconomic status) and poor educational outcomes, have a strong association with both chronic medical and mental health conditions [8]. Furthermore, risk behaviors that are considered modifiable, such as drug and alcohol consumption, lack of physical activity, and poor nutrition, are directly related to chronic medical disease and are often corollaries of mental health conditions (if not mental health conditions themselves, i.e., substance use disorder) [8].

The mechanics of how mental health conditions and medical comorbidities affect individual functioning are understandable from a behavioral point of view. Both mental health and medical symptoms impact psychosocial functioning and/or lead to maladaptive behaviors. According to the Diagnostic and Statistical Manual, $5^{\text {th }}$ Edition (DSM5), diagnostic criteria for most mental health disorders includes a criterion for the presence of "clinically significant distress or impairment in social, occupational, or other important areas of functioning" [14,15]. In other words, to confirm presence of a mental health condition, it must significantly affect someone's day-to-day functioning in their job, relationships, academics, and self-care, and/or it must weaken someone's emotional wellbeing to the point of distress. It can also lead to development of poor coping behaviors, such as isolation and withdrawal or substance use; in an attempt to manage both the condition and increases in stress from impaired functioning. Likewise, impacted mobility from medical conditions can also lead to social isolation and withdrawal, as well as physical inactivity, poor nutrition, and even substance use.

This impairment of functioning and development of maladaptive behaviors is most often seen in the context of adherence to treatment. Generally, treatment adherence is defined as adhering to at least $80 \%$ of a prescribed treatment and/or medication [16]. A comprehensive literature review found that patients' beliefs, motivations, and attitudes (negative) are strongly correlated with healthcare treatment adherence [16]. Beliefs, motivations, and attitudes tend to be severely impacted by depression and anxiety. If those factors, in addition to emotional wellbeing and day-to-day functioning, are impacted, it can be difficult for many chronically ill patients to adhere to their prescribed care plans. Likewise, impaired mobility and dayto-day functioning from chronic medical conditions can induce or exacerbate onset of depression, anxiety, PTSD symptoms, and poor emotional wellbeing $[17,18]$. It can be a vicious cycle. For example, depression symptoms are often associated with fatigue/apathy and withdrawal/social isolation. Fatigue and apathy can impair the self-discipline and effort needed to adhere to treatment recommendations. Lack of adherence can worsen the medical condition, which can exacerbate depression symptoms. Similarly, the maladaptive behavior stemming from anxiety symptoms is avoidance. Avoidance can manifest toward external stimuli as well as internal stimuli, such as emotional states and body sensations. If a treatment plan seems too intimidating or induces anxiety, it can cause someone to avoid into non-adherence. Similar to depression and anxiety, PTSD can involve withdrawal/isolation and avoidance. Non-adherence from depression, anxiety, or PTSD can lead to an unintended stigmatization where the patient is blamed for poor adherence when in fact there are significant mental health obstacles that hinder successful adherence outcomes. Attitudes about adherence perpetuate stereotypes and stigma related to mental health.

The medical conditions of diabetes and cardiovascular 
Citation: Norman KP, Norman SR, Muralidharan M, et al. (2020) Perspectives on the Emerging Digital Transformation of the Management of Psychiatric Comorbidities in Chronically III Populations. J Psychiatry Treat Res 2(1):25-31

disease are good illustrations of this relationship. Diabetes is a particularly important example of this problem since (i) Diabetes is a common illness (1 out of every 11 people worldwide have diabetes) $[19,20]$; (ii) Diabetes has high rates of comorbid depression, anxiety; and PTSD [12,21] and (iii) Effective diabetes management requires patients to self-manage a complex food intake, physical activity, and medication regimen [22]. Thus, adherence to this treatment would require $80 \%$ of those recommendations to be followed [16]. Regarding depression and anxiety, the prevalence rates of depression are up to three times higher in patients with type 1 diabetes, and twice as high in people with type 2 diabetes, compared to the general population $[23,24]$. As a consequence, in patients with type 1 and type 2 diabetes, $25 \%$ are affected by depression; as many as $40 \%$ are affected by anxiety; and those with significant ACEs had 2.3 times the mortality rate of the normal population $[23,25]$. Further, $50 \%$ of all diabetes patients experience Diabetes Distress, which is a disturbed emotional state that does not meet the clinical criteria for anxiety disorder, but nevertheless causes people to experience feelings such as stress, guilt, or denial that arise from living with diabetes and the burden of self-management of their care plan [26-28]. As a result, debilitating mental health conditions make it difficult for $75 \%$ of type 1 and type 2 diabetes patients to adhere to prescribed care plans [2932]. Fatigue and apathy from both depression and diabetes symptoms can make it difficult to adhere to a consistent regimen of food intake, physical activity, and medication. In clinical depression cases without medical comorbidity, it can already be difficult to progress a patient toward behavioral activation, and the difficulty may be cumulative in patients with medical and mental health comorbidities.

Cardiovascular disease (CVD) is another particularly important example of this problem from the standpoint of cardiac self-efficacy. Evidence suggests that self-efficacy is a significant predictor of heart failure hospitalizations [33], and it could further be posited to be associated with treatment adherence. Self-efficacy is a psychological construct that posits that the higher someone's self-confidence in their own ability (self-efficacy) the more likely they are to engage in and perform those behaviors [34]. It has been positively associated with various health outcomes, such as diet, exercise, and chronic disease management [33]. One of the hallmarks of depression, anxiety, and PTSD is reduced self-efficacy: in depression, feelings and beliefs of worthlessness; in anxiety, beliefs of inadequacy or incapacity; in PTSD, both beliefs of worthlessness and inadequacy, along with distressing thoughts and memories and intense physiological reactions. From a Cognitive Behavioral Therapy (CBT) formulation, the beliefs of worthlessness and inadequacy become so ingrained in an individual that their behaviors become an automated derivative of the underlying beliefs. Their worldview becomes a perspective filtered by a lens of reduced self-efficacy, to the extent there is an inability see a higher self-efficacious alternative. Until these beliefs are targeted and challenged in a psychotherapeutic context, they can perpetuate indefinitely. In PTSD in particular, studies have shown that treatment of the underlying mental health condition can improve medical conditions, such as diabetes [35], and a similar effect is hypothesized with CVD [36]. Consequently, if these beliefs stay unaddressed, it increases the probability that treatment will be non-adherent.

\section{The Problem of Treatment Access and Re- sources}

The proverbial "good news...bad news" is that depression and anxiety are among the most treatable of conditions, yet less than $50 \%$ of people with those conditions receive treatment $[37,38]$. A review of meta-analyses indicated strong effects (with some mixed evidence comparative to other interventions) across studies of CBT interventions for depression and anxiety [39]. It is considered the gold standard of psychotherapy [40]. Yet, an estimated 118 million Americans are unable to access effective mental health care because of the critical dearth of available treatment resources and significant barriers to access, which include cost, stigma, distance, and inconvenience $[37,41]$. Otherwise stated, there are evidence-based treatments that work, but there is a problem in connecting those treatments with the people that need them.

On the subject of available treatment resources, reports from the American Association of Medical Colleges (AAMC) predict shortages in medical and mental health clinicians to expand in the next decade [42]. The Health Resources and Services Administrations (HRSA) predicts that even despite increases in supply of mental healthcare workers, demand will continue to exceed supply [43]. Thus, it is unlikely that hospitals and mental health clinics will be able to meet the in-person demand for services [44]. Compounding the problem is the unequal distribution of clinical professionals in urban as opposed to rural areas [44]. In sum, there is an access problem in conjunction with a problem of finding adequate scalability of supply.

Furthermore, for a set of chronic conditions that are so interrelated and co-occur so often, the treatment for medical and mental health conditions is almost always siloed. It is not uncommon for mental health clinicians to stay away from medical issues, pushing them off to medical disease specialists, and vice versa. Rarely is treatment of both conditions integrated. Psychological factors are often unaccounted for in medical conditions, and factors from medical conditions are often unaccounted for in mental conditions. Patients are treated for health conditions in hospital settings without consistent psychological evaluations of level of depression, anxiety etc. because traditional psychotherapy does not have a way to scale innovative tools for providing psycho-education and patient management in primary care settings. The 1:1 model, while effective, does not address the needs from a population health perspective. As an added incentive, medical-mental health integration has the potential to yield significant cost reductions [45]. Furthermore, there is evidence of successful medical and mental health treatment integration from a randomized controlled trial that concurrently targeted type 2 diabetes and depression [46]. However, the 
Citation: Norman KP, Norman SR, Muralidharan M, et al. (2020) Perspectives on the Emerging Digital Transformation of the Management of Psychiatric Comorbidities in Chronically III Populations. J Psychiatry Treat Res 2(1):25-31

implementation of integrated medical and mental health treatment is not yet wide-spread. Hence, there remains a profound lack of integration between medical and mental health.

\section{The Digital Transformation of Mental Well- being}

There is an encouraging potential solution to these problems of access, scalability, and integration. Advancements in technology enable significant increases in access to quality medical and mental health care. According to the Pew Research Center, as of $2018,81 \%$ of Americans own a smart phone, up from $35 \%$ of people in 2011 [47], meaning that a vast majority of people have access to the internet at their fingertips. This alone presents a ripe environment to enable access to mental health care by reaching people where they are. Further, several studies have now shown that CBT administered online can be as effective as in-person psychotherapies [48-52]. However, although online administrations can be effective, there remains a problem with online therapy being engaging enough to result in higher completion rates. In fact, forms of online CBT administration that relied on automated open access, and that were self-paced, tended to suffer low completion rates $[53,54]$. Historically the criticism about digital interventions have centered around difficulty with providing a relational experience that is inherently a part of and comparable to psychotherapy. Thus, a solution that offers the patient a mix of automated and non-automated contact, to provide the relatability that is a part of in-person psychotherapy, and that can integrate social and peer support community as a part of the treatment offers a compelling path forward in digital transformation of mental wellbeing. In other words, a promising end goal of the digital transformation would be a solution that takes the best parts of individual and group psychotherapy, comprehensively addresses the underlying mental health condition, is delivered in a compelling and interesting way, and increases access on a massive scale, thereby increasing engagement and completion.

Administration of CBT and other type interventions by telemedicine means (that is, via telephonic or videoconferencing services) is considered in the first wave of technology-enabled access. Telemedicine enables clinicians to meet with, evaluate, and treat patients over virtually any distance, removing logistical barriers to care. Within mental health, no-show and cancellation rates are particular problems that consume resources without provision of treatment. Evidence suggests that telemedicine has demonstrated success in reducing no-show rates and cancellations [55]. Although telemedicine increases access, it is unable to scale adequately to address the gap in supply and demand of services because it still represents a 1:1 approach to care delivery, i.e., one therapist treating one patient. Thus, the first wave of technology-enabled telemedicine-mediated access has increased the reach of and access to mental health services but has been insufficient at scaling to meet the demand for and integration of mental health services.

The second wave of technology-enabled access are the mobile apps that target specific behavioral changes. In recent years, there has been a massive proliferation and development of e-health and mental health apps [56]. As of 2018, there were over 10,000 mental health apps in the market. According to a mini-review, only around $3 \%$ of mental health apps had research supporting findings; less than one-third had expert clinical professional input; and only around onefifth were associated with an academic institution, medical center, or government organization [56]. Without clinical input in mental health mobile app development or structured and rigorous research to measure effects both short and long term, effectiveness cannot be determined. In other words, current mobile apps target specific mental health condition-related behaviors, such as physical inactivity, poor sleep hygiene, and weight control, yet, the vast majority attempt to treat these conditions without certainty that evidence-based practices are being utilized. Furthermore, despite mass proliferation, usage of mental health apps remains low [57]. Thus, we posit that the second wave addresses isolated, specific behavioral changes without addressing the causative underlying mental health conditions in an evidence-based way. We also posit that current mental health apps tend to be limited in their usability over time. Otherwise stated, they don't grow as the user grows, and thus become generally obsolete. Thus, although the second wave has supplied mental health apps that increase access and scale, they are generally insufficient in quality to adequately fulfill the demand of mental health treatment and are specifically unable to address mental health and medical comorbidity.

We are now beginning to crest the third wave of technology-enabled access to health and wellbeing. This trend is part of the emerging "internet of things" approach to design that has defined the third wave of information technology more broadly, and which seeks to weave the use of interdependent technologies more seamlessly into everyday life [58]. In the third wave of digital behavioral health technologies, this trend is demonstrated by the shift towards addressing the underlying mental health conditions and medical comorbidities by unifying under a single point of access the many factors that influence mental and physical health. This comes in the form of asynchronous administration of evidence-based mental health care services and artificial intelligence-enabled (Al-enabled) technology that increases both the access and scale of mental health services and provides opportunities for chronic disease integration. This includes digital delivery of CBT that would provide reliable access to safe, effective, and affordable mental health care services, and addresses the scalability problem to reach hundreds of millions of people who are currently unserved. This type of delivery would increase engagement by enabling personalized clinical content, optimized by expert clinicians, to meet the needs of specific patient populations and, ultimately, individual users. This form of computerized psychotherapy can be much richer in multimedia content, with text, images, videos, animations, audio voiceovers, and interactive exercises being tailored by clinical and technology experts to deliver a personalized treatment program that offers a very engaging user experience. These interventions can deliver CBT, mindfulness, and psychoeducation in an interesting and compelling way. They provide the opportunity to examine a personal story through 
Citation: Norman KP, Norman SR, Muralidharan M, et al. (2020) Perspectives on the Emerging Digital Transformation of the Management of Psychiatric Comorbidities in Chronically III Populations. J Psychiatry Treat Res 2(1):25-31

narrative and receive personal insights from analysis of narrative content [59], while at the same time facilitating the interpersonal connections that foster both online and real world communities of peer support and accountability. These types of engaging computerized psychotherapies will have the highest probability of effectively enhancing access and drastically improving scale without the attrition rates seen in the first wave of online CBT therapies. Initial evidence of effectiveness of third wave digital delivery is promising, with depression, anxiety, PTSD, and somatic symptoms reducing significantly and retention rates around $75 \%[59,60]$.

Future success in the treatment of comorbid disorders will be directly linked to the ease of integration between the medical field and the mental health/psychiatric field. Similar approaches to integrating mental health services into hypertension, cancer, and diabetes treatment are in early stages of development. The integration of digitally delivered mental health intervention within standard treatments for other chronic disease conditions with elevated rates of co-occurring depression and anxiety (e.g. cerebrovascular diseases [23\% depression/22\% anxiety]; COPD 25\% [depression/44\% anxiety] and obesity treatment [43\% depression/37\% anxiety]) are future opportunities. Digital delivery is a cost-effective way to facilitate these treatment integrations, and the engaged nature of interactive computerization of treatment has the potential to increase treatment adherence across the board.

An example of this comes in a hybrid form of medical rehabilitation that combines in-person and digital delivery of mental health interventions to address issues that may impact treatment adherence. This framework is currently being implemented at a West Coast university that has integrated a digitally delivered mental health intervention within a standard cardiovascular rehabilitation treatment in order to scale mental health services and increase cardiovascular rehabilitation treatment adherence and cardiovascular-specific clinical outcomes. The objective is to reduce the mental health barriers to the patient. If access to mental health treatment is made easy, and that access includes addressing factors that may get in the way of cardiovascular rehabilitation treatment, then it increases the probability of adherence to the cardiovascular rehabilitation; successful cardiovascular disease-specific clinical outcomes; and, ultimately, lower overall costs of cardiovascular care. The potential secondary effects of this understanding of barriers to adherence are reduction of patient stigma and stereotypes. In this way, digital therapeutic tools also offer a scalable opportunity for advocacy and activism in integrated holistic health.

\section{Conclusion}

This third wave of digital delivery of evidence-based mental healthcare programs will open up the possibility that businesses, schools, and even primary care settings can become the opportune point-of-care for mental healthcare and integrated rehabilitation and treatment services. It enables the optimization of content to meet the needs of specific target populations; enables generation of a social community to overcome social isolation; and meets the needs of individual mental healthcare consumers. It solves the problem of integration of treatment for medical and mental health comorbidities, thereby increasing treatment adherence, improving clinical outcomes, and ultimately saving lives and reducing the total cost of disease management. It solves the problem of attrition with mental health services because a highly personalized user experience would drive increased user engagement, so more participants would complete and benefit from their digitally delivered program. Online computerized therapies can be modified at any time to make sure they are using only the latest evidence-based treatment methods, and people can access them from their personal devices whenever and wherever they prefer. Finally, imagine being able to treat, on a massive scale, the underlying traumas that result in high ACE scores, predisposing individuals to variety of chronic medical conditions. The third wave of digital delivery of evidence-based mental healthcare has the capacity to address these traumas and their correlative medical conditions in tandem.

\section{Disclosures}

Dr. Norman serves as an advisor to Tiatros, Inc., compensated by stock options. Dr. Norman has a household financial interest in Tiatros, Inc. Dr. Kieschnick is a project-based contractor for Tiatros, Inc.

\section{References}

1. Raghupathi W, Raghupathi V (2018) An empirical study of chronic diseases in the United States: a visual analytics approach to public health. Int J Environ Res Public Health 15: E431.

2. McGlynn EA, Asch SM, Adams J, et al. (2003) The quality of health care delivered to adults in the United States. N Engl J Med 348: 2635-2645.

3. https://www.nimh.nih.gov/health/statistics/index.shtml.

4. Huang H, Yan P, Shan Z, et al. (2015) Adverse childhood experiences and risk of type 2 diabetes: A systematic review and meta-analysis. Metabolism 64: 1408-1418.

5. Wade TJ, O'Leary DD, Dempster KS, et al. (2019) Adverse childhood experiences (ACEs) and cardiovascular development from childhood to early adulthood: Study protocol of the Niagara Longitudinal Heart Study. BMJ Open 9.

6. Allen Heidi, Wright Bill J, Vartanian Keri, et al. (2019) Examining the prevalence of adverse childhood experiences and associated cardiovascular disease risk factors among low-income uninsured adults. Circ Cardiovasc Qual Outcomes 12: e004391.

7. Alegria M, Jackson JS, Kessler RC, et al. (2016) Collaborative psychiatric epidemiology surveys (CPES), 2001-2003 [United States] (ICPSR 20240). ICPSR Find \& Analyze Data.

8. (2011) Mental disorders and medical comorbidity. RWJF.

9. Celano CM, Daunis DJ, Lokko HN, et al. (2016) Anxiety disorders and cardiovascular disease. Curr Psychiatry Rep 18: 101.

10. Coughlin SS (2011) Post-traumatic stress disorder and cardiovascular disease. Open Cardiovasc Med J 5: 164-170.

11. Hendrickson LCM, Neylan TC, Na B, et al. (2013) Lifetime trauma exposure and prospective cardiovascular events and all-cause mortality: Findings from the heart and soul study. Psychosom Med 75: 849-855. 
Citation: Norman KP, Norman SR, Muralidharan M, et al. (2020) Perspectives on the Emerging Digital Transformation of the Management of Psychiatric Comorbidities in Chronically III Populations. J Psychiatry Treat Res 2(1):25-31

12. Boscarino JA (2004) Posttraumatic stress disorder and physical illness: Results from clinical and epidemiologic studies. Ann N Y Acad Sci 1032: 141-153.

13. Yi JC, Syrjala KL (2017) Anxiety and depression in cancer survivors. Med Clin North Am 101: 1099-1113.

14. American Psychiatric Association (2013) Diagnostic and statistical manual of mental disorders (DSM- $\left.5^{\circ}\right)$. American Psychiatric Pub.

15. Kieschnick D (2019) The impact of coping resources on psychosocial functioning in veterans with multiple comorbidities. ProQuest.

16. Jin J, Sklar GE, Min Sen Oh V, et al. (2008) Factors affecting therapeutic compliance: A review from the patient's perspective. Ther Clin Risk Manag 4: 269-286.

17. Hirvensalo M, Sakari-Rantala R, Kallinen M, et al. (2007) Underlying factors in the association between depressed mood and mobility limitation in older people. Gerontology 53: 173-178.

18. Ostir GV, Ottenbacher KJ, Fried LP, et al. (2007) The effect of depressive symptoms on the association between functional status and social participation. Soc Indic Res 80: 379-392.

19. (2018) Diabetes. WHO.

20. (2019) IDF diabetes atlas 9th edition 2019. International Diabetic Federation.

21. Ducat L, Philipson LH, Anderson BJ (2014) The mental health comorbidities of diabetes. JAMA 312: 691-692.

22. Shrivastava SR, Shrivastava PS, Ramasamy J (2013) Role of selfcare in management of diabetes mellitus. J Diabetes Metab Disord 12: 14.

23. Roy T, Lloyd CE (2012) Epidemiology of depression and diabetes: A systematic review. J Affect Disord 142: S8-S21.

24. Bădescu S, Tătaru C, Kobylinska L, et al. (2016) The association between diabetes mellitus and depression. J Med Life 9: 120125.

25. Campbell JA, Mosley-Johnson E, Garacci E, et al. (2019) The co-occurrence of diabetes and adverse childhood experiences and its impact on mortality in US adults. J Affect Disord 249: 20-25.

26. Nanayakkara N, Pease A, Ranasinha S, et al. (2018) Depression and diabetes distress in adults with type 2 diabetes: Results from the Australian National Diabetes Audit (ANDA) 2016. Sci Rep 8.

27. Fisher L, Gonzalez JS, Polonsky WH (2014) The confusing tale of depression and distress in patients with diabetes: A call for greater clarity and precision. Diabet Med J Br Diabet Assoc 31: 764-772.

28. CDC (2019) Diabetes and mental health. Centers for Disease Control and Prevention.

29. García-Pérez L-E, Álvarez M, Dilla T, et al. (2013) Adherence to therapies in patients with type 2 diabetes. Diabetes Ther 4: 175194.

30. (2003) Adherence to Long-Term Therapies - Evidence for Action: Section III - Disease-Specific Reviews: Chapter X - Diabetes: 4. Prevalence of adherence to recommendations for diabetes treatment.

31. Polonsky WH, Henry RR (2016) Poor medication adherence in type 2 diabetes: Recognizing the scope of the problem and its key contributors. Patient Prefer Adherence 10: 1299-1307.
32. Holt RIG, de Groot M, Golden SH (2014) Diabetes and depression. Curr Diab Rep 14: 491.

33. Sarkar U, Ali S, Whooley MA (2009) Self-efficacy as a marker of cardiac function and predictor of heart failure hospitalization and mortality in patients with stable coronary heart disease: Findings from the Heart and Soul Study. Health Psychol 28: 166173.

34. Bandura A (1977) Self-efficacy: Toward a unifying theory of behavioral change. Psychol Rev 84: 191-215.

35. Scherrer JF, Salas J, Norman SB, et al. (2019) Association between clinically meaningful posttraumatic stress disorder improvement and risk of type 2 diabetes. JAMA Psychiatry 76: 1159-1166.

36. Edmondson D, Cohen BE (2013) Posttraumatic stress disorder and cardiovascular disease. Prog Cardiovasc Dis 55: 548-556.

37. The Doctor is Out | NAMI: National Alliance on Mental Illness.

38. Facts \& Statistics | Anxiety and Depression Association of America, ADAA.

39. Hofmann SG, Asnaani A, Vonk IJJ, et al. (2012) The efficacy of cognitive behavioral therapy: A review of meta-analyses. CognTher Res 36: 427-440.

40. David D, Cristea I, Hofmann SG (2018) Why cognitive behavioral therapy is the current gold standard of psychotherapy. Front Psychiatry 9.

41. New Study Reveals Lack of Access as Root Cause for Mental Health Crisis in America. National Council.

42. (2019) The 2019 Update: The complexities of physician supply and demand: Projections from 2017 to 2032. AAMC.

43. Behavioral Health Workforce Projections, 2016-2030.

44. Olfson M (2016) Building the mental health workforce capacity needed to treat adults with serious mental illnesses. Health Aff (Millwood) 35: 983-990.

45. Melek S, Norris DT, Paulus J (2014) Economic impact of integrated medical behavioral healthcare: Implications for Psychiatry.

46. Bogner HR, Morales KH, Vries HF de, et al. (2012) Integrated management of type 2 diabetes mellitus and depression treatment to improve medication adherence: A randomized controlled trial. Ann Fam Med 10: 15-22.

47. https://www.pewresearch.org/internet/fact-sheet/mobile/

48. Azimi M, Moradi A, Hasani J (2019) Effectiveness of cognitive behavioral therapy for insomnia (traditional and Internet-based) on everyday memory of people with insomnia and comorbid depression. Adv Cogn Sci 20: 20-34.

49. Kuester A, Niemeyer H, Knaevelsrud C (2016) Internet-based interventions for posttraumatic stress: A meta-analysis of randomized controlled trials. Clin Psychol Rev 43: 1-16.

50. Spek V, Cuijpers P, Nyklíček I, et al. (2007) Internet-based cognitive behaviour therapy for symptoms of depression and anxiety: A meta-analysis. Psychol Med 37: 319-328.

51. Spek V, Cuijpers P, Nyklíček I, et al. (2008) One-year follow-up results of a randomized controlled clinical trial on Internet-based cognitive behavioural therapy for subthreshold depression in people over 50 years. Psychol Med 38: 635-639.

52. Simon N, McGillivray L, Roberts NP, et al. (2019) Acceptability of internet-based cognitive behavioural therapy (i-CBT) for post-traumatic stress disorder (PTSD): A systematic review. Eur J Psychotraumatology 10: 1646092. 
Citation: Norman KP, Norman SR, Muralidharan M, et al. (2020) Perspectives on the Emerging Digital Transformation of the Management of Psychiatric Comorbidities in Chronically III Populations. J Psychiatry Treat Res 2(1):25-31

53. Farvolden $P$, Denisoff $E$, Selby $P$, et al. (2005) Usage and longitudinal effectiveness of a web-based self-help cognitive behavioral therapy program for panic disorder. J Med Internet Res 7.

54. Christensen H, Griffiths KM, Korten AE, et al. (2004) A comparison of changes in anxiety and depression symptoms of spontaneous users and trial participants of a cognitive behavior therapy website. J Med Internet Res 6: e46.

55. Leigh H, Cruz H, Mallios R (2009) Telepsychiatry appointments in a continuing care setting: Kept, cancelled and no-shows. J Telemed Telecare 15: 286-289.

56. Marshall JM, Dunstan DA, Bartik W (2019) The digital psychiatrist: In search of evidence-based apps for anxiety and depression. Front Psychiatry 10.
57. Torous J, Wisniewski H, Liu G, et al. (2018) Mental health mobile phone app usage, concerns, and benefits among psychiatric outpatients: Comparative survey study. JMIR Ment Health 5: e11715.

58. Tan L, Wang N (2010) Future internet: The internet of things. $20103^{\text {rd }}$ International Conference on Advanced Computer Theory and Engineering (ICACTE), Chengdu, China.

59. Norman K, Govindjee A, Norman S, et al. Natural Language Processing Tools for Assessing Progress and Outcome of Two Veteran Populations: Insights from a Novel Online Intervention for Post Traumatic Growth (In Review). J Med Internet Res. (In Review).

60. Norman S, Barcella M, Muralidharan M, et al. Evaluation of an Online Resilience Skills Training Program for Employee Wellness. In Review. 